\title{
Diagnostic value of urinary RBP, ALB and AQP2 in neonatal hydronephrosis and the relationship with expression of MCP-1 in the prenatal maternal peripheral blood
}

\author{
MA ZHI ${ }^{1}$, YANAN ZHANG ${ }^{2}$, LIXIA LIU ${ }^{2}$ and HUIZHI WANG ${ }^{2}$ \\ Departments of ${ }^{1}$ Pediatric Surgery and ${ }^{2}$ Obstetrics and Gynecology, \\ Hongqi Hospital Affiliated to Mudanjiang Medical College, Mudanjiang, Heilongjiang 157011, P.R. China
}

Received April 12, 2018; Accepted October 17, 2018

DOI: $10.3892 /$ etm.2018.6913

\begin{abstract}
Diagnostic value of urinary retinol binding protein (RBP), albumin (ALB) and aquaporin-2 (AQP2) in neonatal hydronephrosis and their relationship with the expression of monocyte chemoattractant protein 1 (MCP-1) in the prenatal maternal peripheral blood was investigated. Forty-six child patients with hydronephrosis admitted to Hongqi Hospital Affiliated to Mudanjiang Medical College from December 2016 to November 2017 were selected as the observation group and the control included 46 normal newborn infants. The urinary $\mathrm{RBP}, \mathrm{ALB}, \mathrm{AQP} 2$ and the expression of MCP-1 in the prenatal maternal peripheral blood in the two groups were compared. The diagnostic value of the combination of urinary RBP, ALB and AQP2 for the neonatal hydronephrosis was accessed through the area under curve (AUC). The changes of urinary RBP, ALB and AQP2 of child patients were observed and the correlations between RBP, ALB, AQP2 and MCP-1 were analyzed. The concentrations of RBP and ALB in the observation group were obviously increased compared to those in the control group. The AQP2 concentration in the observation group was lower than that in the control group. In the observation group, the MCP-1 level in the prenatal maternal blood was significantly higher than that in the control group $(\mathrm{P}<0.05)$. After treatment, the concentration of RBP and ALB in the child patients were significantly decreased and AQP2 concentration was increased compared with that before treatment $(\mathrm{P}<0.05)$. The AUC of the diagnosis combining with RBP, ALB and AQP2 was 0.913. RBP and ALB were positively correlated to $\mathrm{MCP}-1$ in the prenatal maternal peripheral blood and there was a negative correlation between AQP2 and MCP-1 ( $<<0.05)$. In conclusion, urinary RBP, ALB and AQP2
\end{abstract}

Correspondence to: Dr Huizhi Wang, Department of Obstetrics and Gynecology, Hongqi Hospital Affiliated to Mudanjiang Medical College, 5 Tongxiang Road, Aimin, Mudanjiang, Heilongjiang 157011, P.R. China

E-mail:w156157158159@163.com

Key words: neonatal hydronephrosis, RBP, ALB, AQP2, MCP-1 can be regarded as markers for the diagnosis of the neonatal hydronephrosis and they are also closely related to the MCP-1 level in the prenatal maternal peripheral blood.

\section{Introduction}

Congenital hydronephrosis is one of the common childhood malformations in clinic which is usually caused by the interaction of glomerular hemodynamics with the changes in renal tubular function, with a morbidity of $1-2 \%$ in newborn infants (1). Hydronephrosis can eventually lead to renal failure in children, so it is very important to find the early congenital hydronephrosis and treat it timely (2). Clinically, the neonatal hydronephrosis is often diagnosed through imaging examination and a variety of assisting biochemical indicators. However, the biochemical indicators such as serum creatinine and blood urea nitrogen are susceptible to various factors. Therefore, the diagnosis often lags and cannot accurately reflect the extent of neonatal renal injury. As a result, looking for indicators reflecting the degree of renal injury of children with hydronephrosis at early stage is necessary (3). Retinol binding protein (RBP) is currently considered to be the most sensitive and stable transporter that reflects the renal function changes in urine and can be monitored at any time for the diagnosis of renal biopsy morphology (4). Urinary micro-albumin (ALB) is one of the early diagnosis markers for the degree of glomerular injury (5). Aquaporin-2 (AQP2) is a kind of aquaporin that widely exists in the special channel for water transporting on the membrane and it is the marker for the diagnosis of hydronephrosis (6). Monocyte chemoattractant protein 1 (MCP-1) is an inflammatory marker whose overexpression would result in the shallow implantation of placenta in pregnant women and the impaired endothelial function, thus bringing an adverse effect on the normal growth of fetuses (7). In the study, the expression levels of urinary RBP, ALB, AQP2 and MCP-1 in the prenatal maternal blood were detected and analyzed to provide the diagnostic proof for neonatal hydronephrosis.

\section{Patients and methods}

Patients. A total of 46 newborn infants with hydronephrosis admitted to Hongqi Hospital Affiliated to Mudanjiang 
Medical College (Mudanjiang, China) from December 2016 to November 2017 were selected as the observation group. Inclusion criteria: i) child patients complying with the hydronephrosis diagnostic criteria (8), ii) child patients who were born within 28 days and iii) approval obtained from the Ethics Committee in Hongqi Hospital Affiliated to Mudanjiang Medical College and informed consent signed by the family members. Exclusion criteria: i) patients with bilateral hydronephrosis and ii) child patients with urinary tract infection and febrile diseases. Compared with the 46 normal newborn infants at the same period in the control group, there were no significant differences in general materials of the child patients in the two groups $(\mathrm{P}>0.05$; Table I).

Methods. The midstream urine samples were collected from two groups of newborn infants in the morning (taken at 6:00 a.m.), and were stored in a refrigerator at $-80^{\circ} \mathrm{C}$ for detection. After removal from the refrigerator during the detection, the urine samples were thawed in water at $4^{\circ} \mathrm{C}$ and centrifuged at $8,100 \mathrm{x} \mathrm{g}$ for $5 \mathrm{~min}$. Neonatal urinary RBP and ALB were detected via the immunity transmission turbidity method. The related kits were provided by Shanghai Beijia Biochemical Reagent Co., Ltd. (Shanghai, China) and the operation was conducted in strict accordance with the instructions. Neonatal urinary AQP2 concentration was detected through the enzymelinked immunosorbent assay (ELISA). The related kits were provided by R\&D systems (Minneapolis, MN, USA) and the determination was in strict accordance with the product manual.

A total of $3 \mathrm{ml}$ of peripheral blood was collected from each pregnant woman in the two groups before the infants were born, placed at $20^{\circ} \mathrm{C}$ for $30 \mathrm{~min}$ and centrifuged at $1,800 \mathrm{x} \mathrm{g}$ for $20 \mathrm{~min}$ at $4^{\circ} \mathrm{C}$. The separated serum was poured into an Eppendorf (EP) tube and stored in a refrigerator at $-80^{\circ} \mathrm{C}$. The serum MCP-1 level was examined via ELISA. The operation was performed strictly according to the protocol of the related kit (provided by Beyotime, Shanghai, China), followed by sample adding, incubation, washing with enzyme, incubation and washing and color development. After the addition of terminative solution, the optical density (OD) value in each well was measured sequentially through a microplate reader (Bio-Rad, Hercules, CA, USA) at the wavelength of $450 \mathrm{~nm}$ within $15 \mathrm{~min}$, and the MCP-1 level was calculated.

\section{Evaluation indexes}

Judgement criteria for the hydronephrosis degree (9): i) mild (renal pelvis and renal calices were full and visualized during 10-30 min, but the cup became shallow), ii) moderate (renal pelvis and renal calices developed during 31-60 min, there were enlargement of renal pelvis and renal calices as well as ureter expansion, but the calices cup disappeared) and iii) severe (renal pelvis and renal calices developed over $60 \mathrm{~min}$ and there was clumpy deformation of renal pelvis and renal calices).

Neonatal urinary $R B P$ and $A L B$ concentrations were detected through immunity transmission turbidity method. Neonatal urinary AQP2 and MCP-1 concentrations in peripheral blood of prenatal pregnant women were detected via ELISA.

Statistical analysis. Statistical Product and Service Solutions (SPSS) 19.0 (SPSS, Inc., Chicago, IL, USA) software was
Table I. Baseline data of child patients in the two groups.

\begin{tabular}{lcccc}
\hline & $\begin{array}{c}\text { Control } \\
\text { group } \\
(\mathrm{n}=46)\end{array}$ & $\begin{array}{c}\text { Observation } \\
\text { group } \\
(\mathrm{n}=46)\end{array}$ & $\mathrm{t} / \chi^{2}$ & P-value \\
Items & $27 / 19$ & $25 / 21$ & 0.044 & 0.833 \\
\hline $\begin{array}{l}\text { Sex (male/female) } \\
\text { Time of birth (days) }\end{array}$ & $6-21$ & $6-23$ & & \\
$\begin{array}{l}\text { Average } \\
\text { day-age (days) }\end{array}$ & $13.58 \pm 5.43$ & $13.83 \pm 5.52$ & 0.219 & 0.827 \\
$\begin{array}{l}\text { Body mass (kg) } \\
\text { Degree of }\end{array}$ & $2.63 \pm 0.76$ & $2.69 \pm 0.75$ & 0.381 & 0.704 \\
hydronephrosis (n, \%) & & & & \\
$\quad \begin{array}{l}\text { Mild } \\
\text { Moderate }\end{array}$ & $29(63.04)$ & $30(65.21)$ & 0.326 & 0.849 \\
Severe & $9(19.57)$ & $7(15.22)$ & & \\
\hline & $8(17.39)$ & $9(19.57)$ & & \\
\hline
\end{tabular}

Table II. Comparison of RBP, ALB AQP2 and maternal MCP-1 levels in the two groups of newborn infants.

\begin{tabular}{|c|c|c|c|c|c|}
\hline Groups & $\mathrm{n}$ & $\begin{array}{c}\mathrm{RBP} \\
(\mathrm{mg} / \mathrm{l})\end{array}$ & $\begin{array}{c}\text { ALB } \\
(\mathrm{mg} / \mathrm{ml})\end{array}$ & $\begin{array}{c}\text { AQP2 } \\
\text { (ng/ml) }\end{array}$ & $\begin{array}{c}\text { Maternal } \\
\text { MCP-1 } \\
(\mathrm{ng} / \mathrm{ml})\end{array}$ \\
\hline $\begin{array}{l}\text { Observation } \\
\text { group }\end{array}$ & 46 & $7.64 \pm 2.63$ & $1.59 \pm 0.78$ & $7.63 \pm 1.24$ & $208.63 \pm 16.43$ \\
\hline $\begin{array}{l}\text { Control } \\
\text { group }\end{array}$ & 46 & $1.96 \pm 0.75$ & $0.37 \pm 0.15$ & $12.79 \pm 2.35$ & $141.22 \pm 13.28$ \\
\hline $\mathrm{t}$ value & & 14.086 & 10.417 & 13.171 & 21.642 \\
\hline P-value & & $<0.001$ & $<0.001$ & $<0.001$ & $<0.001$ \\
\hline
\end{tabular}

RBP, retinol binding protein; ALB, albumin; AQP2, aquaporin-2; MCP-1, monocyte chemoattractant protein 1 .

used for data processing. Measurement data were presented as mean \pm standard deviation, and t-test was used for the examination. Measurement data were shown as ratio, and $\chi^{2}$ test was used for the examination. Diagnostic value was analyzed via receiver operating characteristic (ROC) curve and the correlation was analyzed via Pearson's correlation coefficient. $\mathrm{P}<0.05$ was considered to indicate a statistically significant difference.

\section{Results}

RBP, ALB and maternal MCP-1 levels. As for the conditions of RBP, ALB and maternal MCP-1 in the two groups of newborn infants, the levels of RBP and ALB in the observation group were significantly higher than those in the control group and the concentration of AQP2 in the observation group was lower than that in the control group. The maternal MCP-1 level in the observation group was significantly higher than that in the control group $(\mathrm{P}<0.05$; Table II).

ROC curve for RBP, $A L B$ and $A Q P 2$. ROC curve of the combined diagnosis of RBP, ALB and AQP2 is shown in Fig. 1, with an area under curve (AUC) of 0.913 , sensitivity of $91.5 \%$ and specificity of $89.8 \%$. 


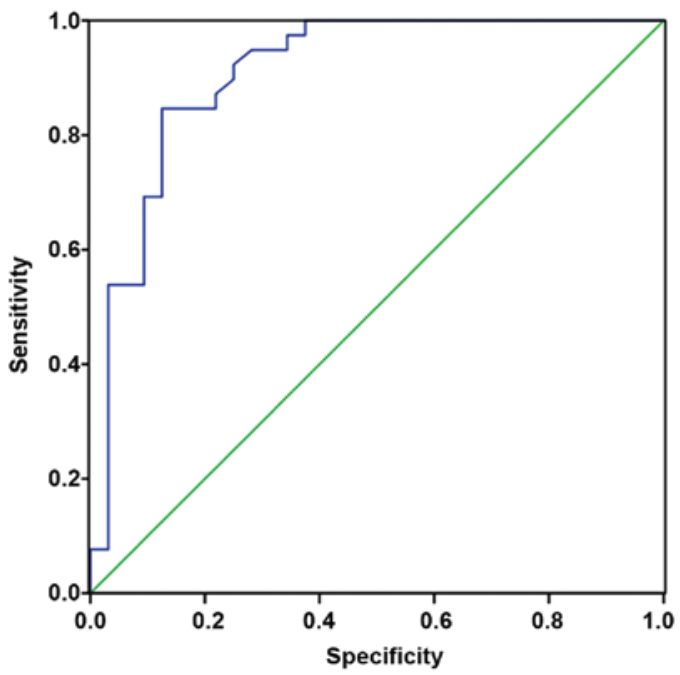

Figure 1. Neonatal hydronephrosis diagnosed through the combination of RBP, ALB and AQP2. The AUC of ROC curve is 0.913 and the sensitivity and specificity are high. RBP, retinol binding protein; ALB, albumin; AQP2, aquaporin-2; AUC, area under curve; ROC, receiver operating characteristic.

Table III. RBP, ALB and AQP2 of child patients before and after treatment.

\begin{tabular}{lcccc}
\hline Time & $\mathrm{n}$ & $\mathrm{RBP}$ & $\mathrm{ALB}$ & $\mathrm{AQP} 2$ \\
\hline Before treatment & 46 & $7.64 \pm 2.63$ & $1.59 \pm 0.78$ & $7.63 \pm 1.24$ \\
After treatment & 46 & $2.56 \pm 1.25$ & $0.68 \pm 0.15$ & $11.89 \pm 1.35$ \\
t value & & 11.832 & 7.770 & 15.762 \\
P-value & & $<0.001$ & $<0.001$ & $<0.001$ \\
\hline
\end{tabular}

RBP, retinol binding protein; ALB, albumin; AQP2, aquaporin-2.

Table IV. Correlation analysis of RBP, ALB and AQP2 with MCP-1.

\begin{tabular}{lcc}
\hline Items & $\mathrm{r}$ & P-value \\
\hline RBP & 0.568 & 0.003 \\
ALB & 0.501 & 0.014 \\
AQP2 & -0.445 & 0.017 \\
\hline
\end{tabular}

RBP, retinol binding protein; ALB, albumin; AQP2, aquaporin-2; MCP-1, monocyte chemoattractant protein 1 .

Changes of RBP, $A L B$ and $A Q P 2$ in child patients before and after treatment. In terms of the changes of RBP, ALB and AQP2 in child patients before and after treatment, the levels of RBP and ALB in child patients after treatment were significantly lower than those before treatment and AQP2 concentration was increased compared with that before treatment $(\mathrm{P}<0.05$; Table III $)$.

The correlation between $R B P, A L B, A Q P 2$ and prenatal $M C P-1$ level. The correlation between RBP, ALB, AQP2 and prenatal MCP-1 level in pregnant women were analyzed

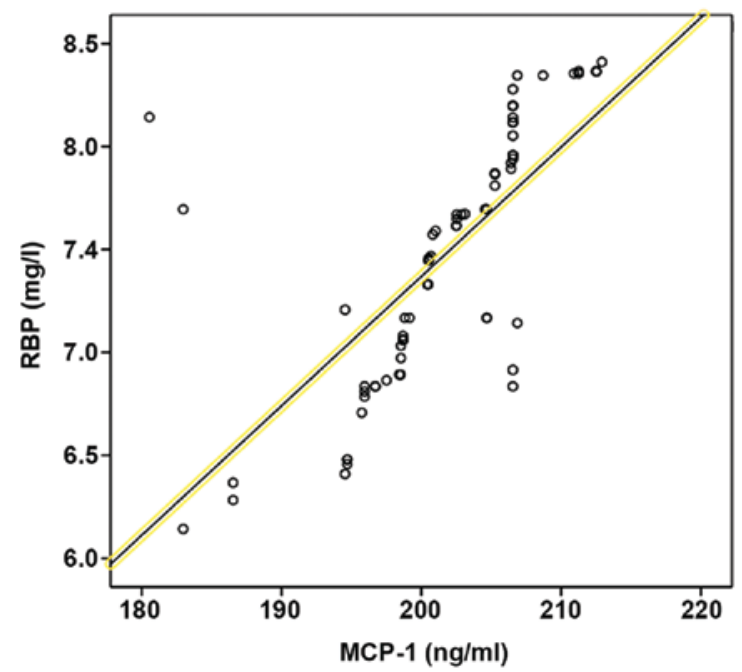

Figure 2. Correlation analysis of RBP with prenatal MCP-1 level in pregnant women. RBP, retinol binding protein; MCP-1, monocyte chemoattractant protein 1 .

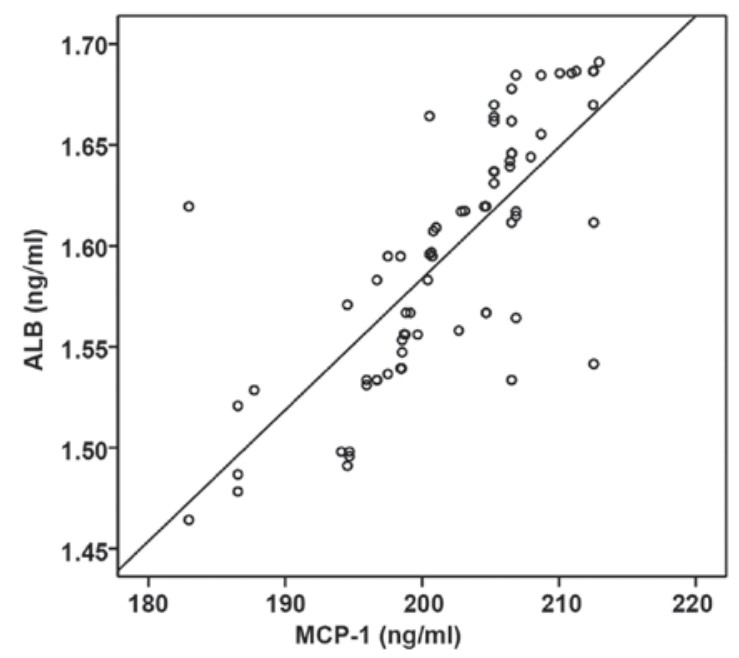

Figure 3. Correlation analysis of ALB with prenatal MCP-1 level in pregnant women. ALB, albumin; MCP-1, monocyte chemoattractant protein 1.

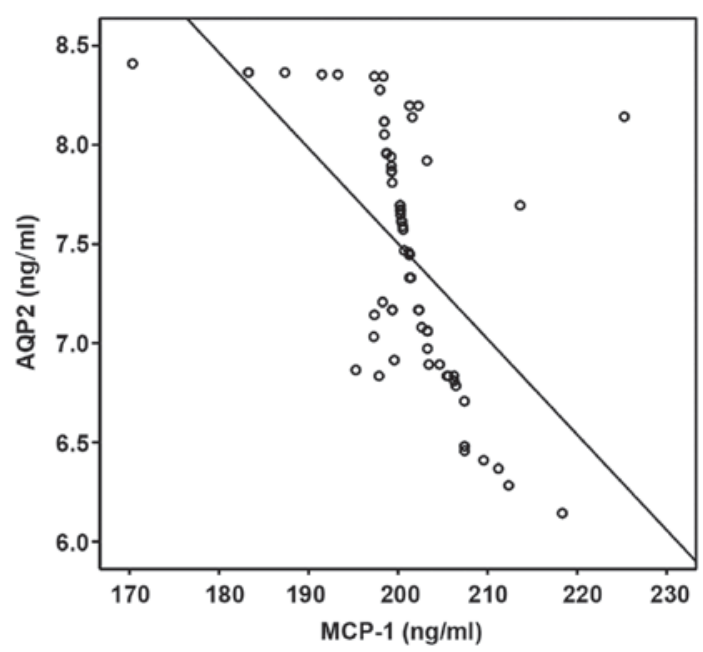

Figure 4. Correlation analysis of AQP2 with prenatal MCP-1 level in pregnant women. AQP2, aquaporin-2; MCP-1, monocyte chemoattractant protein 1 . 
via Pearson's correlation coefficient. RBP and ALB were positively correlated with MCP-1, but AQP2 and MCP-1 were negatively correlated ( $\mathrm{P}<0.05$; Table IV, Figs. $2-4)$.

\section{Discussion}

Neonatal hydronephrosis can account for $>50 \%$ of prenatal defects, which can be caused by the urinary catheter end stenosis, vesicoureteral reflux, posterior urethral valve, ureteral stenosis and ureterocele. Most of the neonatal hydronephrosis results from the congenital ureteral renal pelvis junction obstruction, accounting for approximately $85 \%$ (10). The urinary tract obstruction leads to increasing pressure in the ureter and renal tubular and brings direct or indirect damage to the renal tubular and renal parenchyma. The manifestations are weakened reabsorption function in the renal tubular, the damaged secretion function of hydrogen ion and potassium ion, decreased urine concentration and abnormal renal function in the distal segment of the kidney (11). With the progress of the disease, the progressive decline of renal function eventually leads to renal failure (12). At present, the diagnosis of hydronephrosis mainly relies on imaging and technology is very advanced. However, the routine detection such as serum creatinine and blood urea nitrogen test for neonates, especially for the early diagnosis of renal damage, is often laging. Surgery should be performed when children's renal function begins to deteriorate. Therefore, timely and dynamic diagnosis of changes in renal function is very important.

RBP is a low molecular weight protein secreted by hepatocytes and belongs to the retinol transporters. It is widely present in body fluids such as blood, urine and cerebrospinal fluid and plays an important role in the storage, transportation and metabolism of vitamin A (13). In clinic, RBP is often used as a marker of renal tubular injury. It is simple to be detected, ready to be monitored and difficult to be decomposed in urine. With strong stability, it is not affected by the $\mathrm{PH}$ value of sphygmomanometer and other factors. Therefore, it can provide effective supplement for the renal biopsy diagnosis (14). Results of this study showed that RBP level in children with hydronephrosis was significantly higher than that in normal children, and it was significantly decreased after treatment $(\mathrm{P}<0.05)$. This is because the glomerular filtration in neonates with hydronephrosis decreases while RBP continues to accumulate. When the renal tubular reabsorption function is impaired, RBP cannot be reabsorbed and degraded, thus it would be excreted with the urine. Then its concentration in urine may increase. After effective treatment, the renal function can recover effectively, RBP is absorbed and degraded again, and therefore, the concentration is naturally decreased in urine.

ALB is a highly sensitive middle-molecule protein that is a marker of glomerular injury (15). Results of this study showed that the level of ALB in children with hydronephrosis was significantly higher than that in normal children, and the level of ALB was significantly decreased after treatment $(\mathrm{P}<0.05)$. This is because ALB is reabsorbed by proximal convoluted tubule in normal people, while in neonates with hydronephrosis, the glomerular filtration rate is decreased, so that the filtration of ALB far exceeds the maximum absorption amount of renal tubular. As a result, ALB enters into the urine and its content increases, indicating that the early glomerular lesions occur where there is some damage. Through the intervention treatment, ALB concentration in the urine can be reduced.

AQP2 was first discovered in the early 1990s, it is a member of the aquaporin family (16). AQP2 mainly exists in the cytoplasm of principal cell of renal collecting duct and the cell membrane towards the luminal side, which can increase the ability of water reabsorption and urine concentration (17). It was indicated in the results of this study that ALB level in children with hydronephrosis was significantly lower than that in normal children and it was significantly increased after treatment $(\mathrm{P}<0.05)$. This is due to the fact that when hydronephrosis occurs in the neonates, the concentration of AQP2 which carries on the trans-membrane transportation of water is decreased, so that the water permeability of collecting ductis also decreased. As a result, the transmembrane transportation of water is hindered and the urine concentration is decreased (18). After effective treatment, the amount of AQP2 on the cell membrane is increased, leading to an increase in water reabsorption.

MCP-1, a chemokine most closely associated with pregnancy, plays a key role in maintaining mononuclear phagocyte localization and the invasion of vascular endothelial trophoblasts (19). Results of this study revealed that the level of MCP-1 in maternal peripheral blood in the observation group was significantly higher than that in the control group $(\mathrm{P}<0.05)$. The reason may be that pregnant women with overexpression of MCP-1 may have shallow implantation of placenta, thus affecting the normal healthy growth of fetuses and increasing the risk of hydronephrosis after birth of the fetuses. In this study, Pearson's correlation coefficient analysis indicated that VRBP and ALB had a positive correlation with MCP-1 while AQP2 and MCP-1 were negatively correlated $(\mathrm{P}<0.05)$. This may be related to the fact that MCP-1 endows chemotaxis on a large number of inflammatory cells and expand the inflammatory response. When the level of MCP-1 content is changed, especially in the case of its constantly increasing concentration, the trophoblast invasion function is affected, and the microvessel density changes occur and lead to placental atherosclerosis, thus causing a series of pathological reactions such as elevated blood pressure (20). Substance exchange between mother and baby is decreased and fetal hypoxia appears, which changes the growth and development. This leads to kidney damage and hydronephrosis, increased RBP and ALB levels and decreased AQP2 level, indicating that there is a positively and negatively dependent relationship between the three indexes and MCP-1.

In conclusion, urinary RBP, ALB and AQP2 can be used as diagnostic indicators for the degree of renal dysfunction, which are closely related to the expression of MCP-1 in the peripheral blood of pregnant women, and can be combined with imaging to guide the diagnosis and treatment of neonatal hydronephrosis.

\section{Acknowledgements}

Not applicable.

\section{Funding}

No funding was received. 


\section{Availability of data and material}

The datasets used and/or analyzed during the present study are available from the corresponding author on reasonable request.

\section{Authors' contributions}

MZ wrote the manuscript and collected the midstream urine samples. YZ detected and analyzed neonatal urinary RBP and ALB concentrations. LL performed ELISA. HW analyzed the changes of RBP. All authors have read and approved the final manuscript.

\section{Ethics approval and consent to participate}

The study was approved by the Ethics Committee of Hongqi Hospital Affiliated to Mudanjiang Medical College (Mudanjiang, China) and informed consents were signed by the parents of the child patients.

\section{Patient consent for publication}

Not applicable.

\section{Competing interests}

The authors declare that they have no competing interests.

\section{References}

1. Masarwa I, Bahouth Z and Halachmi S: Giant congenital hydronephrosis obstructing the gastrointestinal system and the contralateral kidney in a new born. Urol Case Rep 8: 1-3, 2016.

2. Meyerholz DK and Hostetter SJ: Unilateral perinephric pseudocyst secondary to hydronephrosis in a C57BL/6J mouse. Vet Pathol 42: 496-498, 2005

3. Al-Mashhadi A, Nevéus T, Stenberg A, Karanikas B, Persson AE, Carlström M and Wåhlin N: Surgical treatment reduces blood pressure in children with unilateral congenital hydronephrosis. J Pediatr Urol 11: 91.e1-91.e6, 2015.

4. Larson LM, Namaste SM, Williams AM, Engle-Stone R, Addo OY, Suchdev PS, Wirth JP, Temple V, Serdula M and Northrop-Clewes CA: Adjusting retinol-binding protein concentrations for inflammation: Biomarkers Reflecting Inflammation and Nutritional Determinants of Anemia (BRINDA) project. Am J Clin Nutr 106 (Suppl 1): 390S-401S, 2017.

5. Gong XZ, Zhou LF, Wang Q, Tang XC, Qian YR, Wang YR, $\mathrm{Lu} \mathrm{L}$ and Zhou JJ: Effect of Chuanhuang No. 1 recipe on renal function and micro-inflammation in phase 3 chronic kidney disease patients. Zhongguo Zhong Xi Yi Jie He Za Zhi 35: 137-141, 2015 (In Chinese).

6. Cui X, Zhang J, Li Y, Sun Y, Cao J, Zhao M, Zhao Y, Zhao X, $\mathrm{He} \mathrm{Y}$ and Han A: Effects of qili qiangxin capsule on AQP2, V2R, and AT1R in rats with chronic heart failure. Evid Based Complement Alternat Med 2015: 639450, 2015.
7. Satonaka H, Nagata D, Takahashi M, Kiyosue A, Myojo M, Fujita D, Ishimitsu T, Nagano T, Nagai R and Hirata Y: Involvement of P2Y12 receptor in vascular smooth muscle inflammatory changes via MCP-1 upregulation and monocyte adhesion. Am J Physiol Heart Circ Physiol 308: H853-H861, 2015.

8. Santos AI, Violante L, Carmona S, Prata A, Rodrigues Victor M, Santos JG, Araújo Sequeira J, Alves M, Papoila AL and Piepsz A: Interobserver agreement on cortical tracer transit in 99mTc-MAG3 renography applied to congenital hydronephrosis. Nucl Med Commun 38: 124-128, 2017.

9. Harding LJ, Malone PS and Wellesley DG: Antenatal minimal hydronephrosis: Is its follow-up an unnecessary cause of concern? Prenat Diagn 19: 701-705, 1999.

10. Zhao Q, Yang Y, Wang C, Hou Y and Chen H: ATP5B and ETFB metabolic markers in children with congenital hydronephrosis. Mol Med Rep 14: 5111-5115, 2016.

11. Vemulakonda VM, Wilcox DT, Torok MR, Hou A, Campbell JB and Kempe A: Inter-rater reliability of postnatal ultrasound interpretation in infants with congenital hydronephrosis. Int Urol Nephrol 47: 1457-1461, 2015.

12. Xie J, Zhou Y, Gao W, Li Z, Xu Z and Zhou L: The relationship between amniotic fluid miRNAs and congenital obstructive nephropathy. Am J Transl Res 9: 1754-1763, 2017.

13. Zabetian-Targhi F, Mahmoudi MJ, Rezaei N and Mahmoudi M: Retinol binding protein 4 in relation to diet, inflammation, immunity, and cardiovascular diseases. Adv Nutr 6: 748-762, 2015.

14. Gursoy AY, Aynaoglu G, Caglar GS and Soylemez F: Early second trimester retinol-binding protein-4 values in cases with or without gestational diabetes mellitus risk factors: A cross-sectional study. J Obstet Gynaecol Res 41: 55-61, 2015.

15. Woo HD, Chiu WA, Jo S and Kim J: Benchmark dose for urinary cadmium based on a marker of renal dysfunction: A metaanalysis. PLoS One 10: e0126680, 2015.

16. Cheema MU, Irsik DL, Wang Y, Miller-Little W, Hyndman KA, Marks ES, Frøkiær J, Boesen EI and Norregaard R: Estradiol regulates AQP2 expression in the collecting duct: A novel inhibitory role for estrogen receptor $\alpha$. Am J Physiol Renal Physiol 309: F305-F317, 2015.

17. Kristensen ML, Kierulf-Lassen C, Nielsen PM, Krag S, Birn H, Nejsum LN and Nørregaard R: Remote ischemic perconditioning attenuates ischemia/reperfusion-induced downregulation of AQP2 in rat kidney. Physiol Rep 4: 4, 2016.

18. Arystarkhova E, Bouley R, Liu YB and Sweadner KJ: Impaired AQP2 trafficking in Fxyd1 knockout mice: A role for FXYD1 in regulated vesicular transport. PLoS One 12: e0188006, 2017.

19. Dekker Nitert M, Barrett HL, Denny KJ, McIntyre HD and Callaway LK; BAMBINO group: Exercise in pregnancy does not alter gestational weight gain, MCP-1 or leptin in obese women. Aust N Z J Obstet Gynaecol 55: 27-33, 2015.

20. Adams Waldorf KM, Singh N, Mohan AR, Young RC, Ngo L, Das A, Tsai J, Bansal A, Paolella L, Herbert BR, et al: Uterine overdistention induces preterm labor mediated by inflammation: Observations in pregnant women and nonhuman primates. Am J Obstet Gynecol 213: 830.e1-830.e19, 2015.

This work is licensed under a Creative Commons Attribution-NonCommercial-NoDerivatives 4.0 International (CC BY-NC-ND 4.0) License. 